\title{
National results and 6 years trends of nosocomial infection surveillance in French intensive care units (REA-RAISIN network)
}

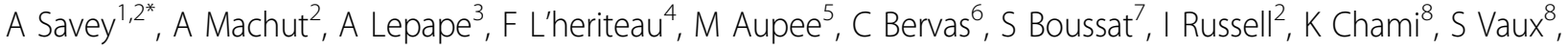 \\ REA-Raisin Network
}

From 3rd International Conference on Prevention and Infection Control (ICPIC 2015)

Geneva, Switzerland. 16-19 June 2015

\section{Introduction}

Healthcare associated infections surveillance is a priority in intensive care units (ICUs).Since 2004, French national surveillance in adult ICUs (REA-RAISIN network) has targeted device-associated infections, for which control measures are essential.

\section{Objectives}

To present 2014 national results and highlight the impact of prevention programmes over the last six years.

\section{Methods}

Six months a year, ICUs collected data for each patient hospitalised more than 2 days. Surveillance focused on ventilation associated pneumonia (VAP), central venous catheter related infection or bacteraemia (CRI/ CRB) and bloodstream infection (BSI) according to European protocol (ECDC). Analysis included patient's characteristics, device exposures, ICU-acquired infections (microorganisms, antimicrobial resistance) and incidence ratios including ICU distributions and temporal trends (2009-2014). Multivariate analyses introducing the year of participation as a risk factor were performed for VAP and CRB.

\section{Results}

In 2014, 212 ICUs included 34,226 patients and 10.7\% presented at least one infection. Overall incidence rates were calculated: $\mathbf{1 4 . 2 6} \mathrm{VAP} / 1,000$ intubation-days, $\mathbf{3 . 5 3}$ BSI/1,000 ICU-days, 0.66 CRI and 0.51 CRB/1,000 catheter-days.

${ }^{1}$ UMR 5558, CNRS - UCBL1, France

Full list of author information is available at the end of the article
In comparison with 2009, patients were more predisposed to infections due to significant evolution of their characteristics (age, SAPSII, antibiotic treatment at admission, immunosuppression) meanwhile device exposure decreased. All incidence rates of device-associated infections decreased significantly: VAP (-6.2\%), CRI (-40.5\%) and CRB (-43.3\%), meanwhile decrease is not significant for BSI (-1.1\%). Multivariate analysis confirmed this reduction in 2014 for VAP (adjusted OR: 0.90; $\mathrm{CI}_{95}: 0.84-0.97$ ) and CRB (adjusted OR: 0.56; $\mathrm{CI}_{95}$ : 0.44-0.71).

\section{Conclusion}

This surveillance network, including $50.4 \%$ of French ICU beds, represents a national reference and appears effective in describing and monitoring infectious risk in ICUs. The significant decrease in 2014 for VAP and CRB can be related to practice improvement and higher level of patient safety.

\section{Disclosure of interest}

None declared.

\section{Authors' details}

${ }^{1}$ UMR 5558, CNRS - UCBL1, France. ${ }^{2}$ CClin Sud-Est, France. ${ }^{3}$ GH Lyon Sud / $\mathrm{HCL}$, Lyon, France. ${ }^{4} \mathrm{CClin}$ Paris-Nord, Paris, France. ${ }^{5} \mathrm{CClin}$ Ouest, Rennes, France. ${ }^{6} \mathrm{CClin}$ Sud-Ouest, Bordeaux, France. ${ }^{7} \mathrm{CCl}$ in Est, Nancy, France. ${ }^{8}$ Institut de Veille Sanitaire, Saint Maurice, France.

Published: 16 June 2015
doi:10.1186/2047-2994-4-S1-P236

Cite this article as: Savey et al:: National results and 6 years trends of nosocomial infection surveillance in French intensive care units (REARAISIN network). Antimicrobial Resistance and Infection Control 2015 4(Suppl 1):P236. 